\title{
Impact of Total Ischemic Time on the Recovery of Regional Wall Motion Abnormality after STEMI in the Modern Reperfusion Era
}

\author{
Jeong Hun Seo, ${ }^{1}$ Kang Hee Kim, ${ }^{2}$ Kwang-Jin Chun, ${ }^{1}$ Bong-Ki Lee, ${ }^{1}$ Byung-Ryul Cho, ${ }^{1}$ \\ and Dong Ryeol Ryu $\mathbb{D}^{1}$ \\ ${ }^{1}$ Division of Cardiology, Department of Internal Medicine, Kangwon National University Hospital, \\ Kangwon National University School of Medicine, Chuncheon-Si, Gangwon-Do, Republic of Korea \\ ${ }^{2}$ Department of Internal Medicine, Kangwon National University Hospital, Chuncheon-Si, Gangwon-Do, Republic of Korea
}

Correspondence should be addressed to Dong Ryeol Ryu; rdr0203@gmail.com

Received 9 November 2021; Revised 14 December 2021; Accepted 6 January 2022; Published 22 January 2022

Academic Editor: Yuichiro Maekawa

Copyright ( $\odot 2022$ Jeong Hun Seo et al. This is an open access article distributed under the Creative Commons Attribution License, which permits unrestricted use, distribution, and reproduction in any medium, provided the original work is properly cited.

Background. Total ischemic time (TIT) is an important factor for predicting mortality among patients with ST-segment elevation myocardial infarction (STEMI). However, the correlation between TIT and the extent of wall motion abnormality has not been well studied. Therefore, we investigated changes in the wall motion score index (WMSI) value based on TIT in STEMI patients who underwent primary percutaneous coronary intervention (PCI) and subsequent transthoracic echocardiography. Methods. STEMI patients who underwent primary PCI and follow-up coronary angiography were analyzed after the exclusion of cases of instent restenosis (ISR). WMSI values were calculated by dividing the sum of scores by the number of segments visualized. Results. A total of 189 patients underwent primary PCI for STEMI, and 151 had no ISR with a median follow-up of 12.3 months. TIT was 180 (117-369) minutes in a subset of 151 patients (mean age of 62 years; $76 \%$ male). Among patients without ISR, 109 (72\%) demonstrated a decrease in the WMSI value during the follow-up period. The WMSI values of patients with TITs of 180 minutes or less were significantly decreased relative to those among patients with TITs of greater than 180 minutes $(p=0.020)$. Among patients with TITs of 180 minutes or less, the TIT was significantly shorter among those with a reduction in the WMSI value than among those with an increase in the WMSI value (106 [81-124] vs. 133 [100-151] minutes; $p=0.018)$. TIT was an independent predictor for a reduction in the WMSI value among these patients (adjusted hazard ratio: $0.976(0.957-0.995) ; p=0.016)$. Conclusions. In the modern reperfusion era of STEMI, patients with TITs of 180 minutes or less experienced a significant degree of recovery from regional wall motion abnormalities.

\section{Introduction}

Timely primary percutaneous coronary intervention (PCI) is the current preferred strategy to treat ST-segment elevation myocardial infarction (STEMI) [1]. The maximum myocardial salvage gained from reperfusion therapy is generally accepted to occur within the first few hours of symptom onset, and the potential for salvaging myocardium is considered minimal or absent after this time. [2,3]. Although the data remain somewhat controversial, the bulk of the evidence also suggests that prolonged total ischemic times (TITs), times from symptom onset to balloon inflation, are associated with an increased risk of mortality $[4,5]$.
The longer the artery is occluded, the more the wavefront of ischemia extends radially from the endocardium to the epicardium [6]. Importantly, the transmural extent and overall size of the infarction are independently predictive of cardiac prognosis in the longer term [7]. Several studies have provided evidence that primary PCI improves left ventricular (LV) dysfunction and inhibits postinfarction dilation and remodeling $[8,9]$. However, little data are available concerning the correlation between TIT and the extent of wall motion abnormality. In addition, although shorter TITs are better, the limitations of the optimal TIT are unclear. Therefore, we sought to investigate changes in the wall motion score 
index (WMSI) value based on the TIT in STEMI patients who underwent primary PCI and subsequent transthoracic echocardiography (TTE).

\section{Methods}

2.1. Study Population. We identified all patients with STEMI who underwent primary PCI between September 2010 and September 2020 at a single center. Among these patients, we screened patients who underwent both follow-up coronary angiography (CAG) and TTE. Patients who experienced death in or out of the hospital as well as those with follow-up loss, only CAG or TTE performed at follow-up, or in-stent restenosis (ISR) were excluded. A total of 151 patients were finally analyzed in this study (Figure 1) and were stratified as those with a decrease and those with an increase in the WMSI value during the follow-up period. This study was approved by the Kangwon National University Hospital Institutional Ethics Committee/Review Board (Study no. 2018-12-004). Informed consent was waived because of the retrospective nature of the study.

2.2. Clinical Data Collection. Demographic and clinical characteristics of patients with STEMI, including age, sex, medical history, Killip class, peak cardiac troponin I and creatine kinase-MB (CK-MB) isoenzyme levels, lipid profiles, and serum creatinine and hemoglobin concentrations, were recorded. Angiographic characteristics of interest included the number of diseased vessels and culprit lesions. In addition, data on medical therapies administered during hospitalization were collected.

2.3. STEMI and Primary PCI. STEMI was diagnosed and treated in accordance with the most recent guidelines $[10,11]$. Briefly, STEMI was defined as a new ST-segment elevation of greater than $0.1 \mathrm{mV}$ on at least two contiguous electrocardiographic leads or the presence of left bundle branch block in patients with acute myocardial infarction. Experienced interventional cardiologists performed primary PCI in all eligible patients with STEMI. Routine medical therapies administered during the perioperative period consisted of aspirin, P2Y12 inhibitors (e.g., clopidogrel, prasugrel, ticagrelor), heparin (either low molecular weight or conventional), and/or glycoprotein IIb/IIIa receptor antagonists. In addition, secondary preventive therapies, including statins, $\beta$-blockers, angiotensin-converting enzyme inhibitors, or angiotensin receptor blockers, were indicated for all patients with STEMI in the absence of contraindications.

2.4. TIT. The TIT was defined as the time from the onset of chest pain to the first occurrence of balloon inflation during primary PCI. This period consisted of onset-to-door and door-to-balloon times. Onset-to-door time was defined as the time from symptom onset to emergency department arrival, whereas door-to-balloon time was the time from emergency department arrival to the first instance of balloon inflation. Information on the timing of symptom onset was obtained by patient interviews. The times of emergency department arrival and first balloon inflation were obtained from patients' medical records.

2.5. Echocardiography and WMSI. Comprehensive TTE was performed on commercially available equipment (Vivid E9; GE Healthcare, Milwaukee, WI, USA or Acuson SC2000; Siemens Medical Solutions, Mountain View, CA, USA). Standard M-mode, two-dimensional, and color Doppler imaging were performed in parasternal, suprasternal, substernal, and apical views with positional adjustment of the patients. The initial TTE was performed within 24 hours after primary PCI. The initial and follow-up echocardiograms recorded during the study period were used to evaluate echocardiographic changes. The median interval of echocardiography was 12.3 months [interquartile range (IQR): 12.1-13.6 months]. Anatomic measurements were made according to the American Society of Echocardiography (ASE) guidelines [12]. WMSI values were obtained by dividing the LV into 16 segments from multiple short-axis views, and apical two-, four-, and long-axis views. This 16segment model consists of six segments at both the basal and mid-ventricular levels and four segments at the apex. The attachment of the right ventricular wall to the left ventricle defines the septum, which is divided at basal and mid-left ventricular levels into anteroseptum and inferoseptum. Continuing counterclockwise, the remaining segments at both basal and mid-ventricular levels are labeled as inferior, inferolateral, anterolateral, and anterior. The apex includes septal, inferior, lateral, and anterior segments. Each segment was analyzed individually and scored on the basis of its motion and systolic thickening. Each of the segments was assigned a score based on the degree of myocardial thickening as follows: one point, normally contracting segment (or hyperkinetic segment); two points, hypokinesis; three points, akinesis; four points, dyskinesis; and five points, aneurysm. The WMSI value was calculated by dividing the sum of scores by the number of segments visualized. Left ventricular measurements and wall motion abnormalities of all patients were reviewed by 2 independent cardiologists. The observers were blinded to one another's WMSI measurements and the clinical endpoint.

2.6. Statistical Analysis. Continuous variables were reported as mean \pm standard deviation values and were compared using Welch's $t$-test or the Wilcoxon rank-sum test, as appropriate. Categorical variables were summarized by frequencies or percentages and analyzed with the chisquared test or Fisher's exact test, as appropriate. Changes in the WMSI value were analyzed with the paired $t$-test. Stepwise multiple linear regression analysis was used to identify the independent predictors of reductions in the WMSI value. All statistical tests were two-tailed, and $p$ values of less than 0.05 were considered statistically significant. All analyses were performed using the Statistical Package for the Social Sciences version 25.0 statistical software (IBM Corporation, Armonk, NY, USA). 


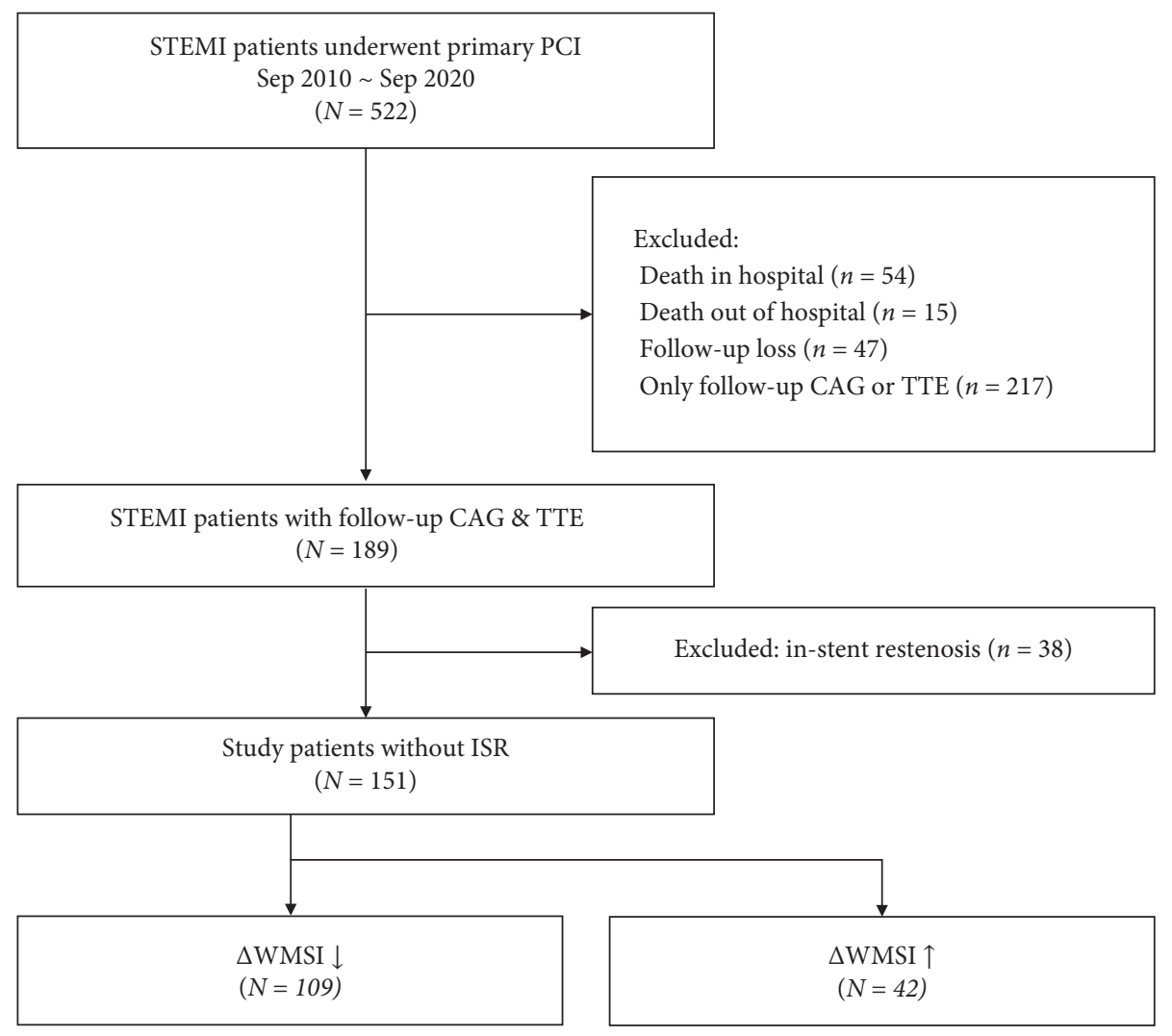

FIGURE 1: Patient flowchart. CAG = coronary angiography; ISR = in-stent restenosis; STEMI = ST-segment elevation myocardial infarction; $\mathrm{TTE}=$ transthoracic echocardiography; WMSI $=$ wall motion score index.

\section{Results}

3.1. Baseline Characteristics and Echocardiography. Baseline characteristics of the patients according to changes in WMSI value are summarized in Table 1 . There were no significant differences in terms of age, sex, comorbidities (e.g., diabetes and hypertension), STEMI location, or medication use between patients who experienced a decrease in WMSI value and those who showed an increase in the WMSI value. The total door-to-balloon time and TIT were statistically indifferent between the two groups. However, there were more patients with TITs of 120 minutes or less among those with a decrease in the WMSI value than among those with an increase in the WMSI value (37 (34\%) vs. $7(17 \%) ; p=0.036)$. Among the laboratory findings, peak CK-MB was lower in the group of patients with WMSI value reductions. The LV end-diastolic dimension was greater in patients with a decrease in the WMSI value than those with an increase in the WMSI value (Table 2).

3.2. Changes in WMSI Value Based on TIT. Figure 2 shows the changes in the WMSI value according to TIT. There were no significant changes in the WMSI value between the two groups based on TITs of 120 and 210 minutes; however, WMSI values in patients with TITs of 150 minutes or less and 180 minutes or less were significantly decreased relative to those of patients with TITs of greater than 150 minutes and greater than 180 minutes ( $p=0.049$ and $p=0.020$, respectively).
3.3. Prediction of WMSI Value Reduction in Patients with TITs of 180 Minutes or Less. In patients with TITs of 180 minutes or less, the TIT was significantly shorter among those with a reduction in the WMSI value than among those with an increase in the WMSI value (106 [81-124] vs. 133 [100-151] minutes, $p=0.018$ ) (Table 3 ). In multivariate logistic regression analysis adjusted for age, sex, body mass index, diabetes, hypertension, and smoking status, TIT was an independent predictor for a decrease in the WMSI value among these patients (adjusted hazard ratio: 0.976 (0.957-0.995); $p=0.016$ ) (Table 4).

\section{Discussion}

In this study, we evaluated changes in the WMSI value based on TIT in STEMI patients who underwent primary PCI and subsequent transthoracic echocardiography. The major findings of this study were as follows: (1) WMSI values in patients with TITs of 180 minutes or less were significantly decreased relative to those in patients with TITs of greater than 180 minutes during the follow-up period; (2) in patients with TITs of 180 minutes or less, the TIT was significantly shorter among the subset with a decrease in the WMSI value than those with an increase in the WMSI value; and (3) TIT was an independent predictor for recovery from regional wall motion abnormalities in patients with TITs of 180 minutes or less. 
TABLE 1: Baseline characteristics.

\begin{tabular}{|c|c|c|c|}
\hline & DWMSI $\downarrow(n=109)$ & $\operatorname{DWMSI} \uparrow(n=42)$ & $p$ value \\
\hline \multicolumn{4}{|l|}{ Demographics } \\
\hline Age, years & $62 \pm 13$ & $61 \pm 10$ & 0.565 \\
\hline Male & $82(75)$ & $33(79)$ & 0.666 \\
\hline Body mass index, $\mathrm{kg} / \mathrm{m}^{2}$ & $24.1 \pm 3.0$ & $24.5 \pm 3.0$ & 0.484 \\
\hline \multicolumn{4}{|l|}{ Medical history } \\
\hline Diabetes & $36(33)$ & $12(29)$ & 0.598 \\
\hline Hypertension & $52(48)$ & $24(57)$ & 0.299 \\
\hline Smoking ever & $52(48)$ & $21(50)$ & 0.800 \\
\hline Previous stroke & $6(5.5)$ & $1(2.4)$ & 0.413 \\
\hline Previous PCI & $7(6.4)$ & $0(0.0)$ & 0.093 \\
\hline \multicolumn{4}{|l|}{ Clinical presentation } \\
\hline Preinfarction angina & $7(6.4)$ & $4(9.5)$ & 0.511 \\
\hline \multicolumn{4}{|l|}{ STEMI location } \\
\hline Anterior & $57(52)$ & $13(31)$ & \multirow{4}{*}{0.075} \\
\hline Inferior & $44(40)$ & $24(57)$ & \\
\hline Lateral & $5(4.6)$ & $5(12)$ & \\
\hline Posterior & $2(1.8)$ & $0(0.0)$ & \\
\hline \multicolumn{4}{|l|}{ Killip class } \\
\hline I & $74(68)$ & $32(76)$ & \multirow{4}{*}{0.521} \\
\hline II & $17(16)$ & $7(17)$ & \\
\hline III & $5(4.6)$ & $1(2.4)$ & \\
\hline IV & $13(12)$ & $2(4.8)$ & \\
\hline \multicolumn{4}{|l|}{ Culprit vessels } \\
\hline Left main coronary artery & $1(0.9)$ & $0(0.0)$ & \multirow{4}{*}{0.010} \\
\hline Left anterior descending artery & $69(63)$ & $15(36)$ & \\
\hline Left circumflex artery & $5(4.6)$ & $6(14)$ & \\
\hline Right coronary artery & $34(31)$ & $21(50)$ & \\
\hline \multicolumn{4}{|l|}{ Extent of CAD } \\
\hline $1 \mathrm{VD}$ & $86(79)$ & $38(91)$ & \multirow{3}{*}{0.222} \\
\hline $2 \mathrm{VD}$ & $20(18)$ & $3(7.1)$ & \\
\hline 3VD & $3(2.8)$ & $1(2.4)$ & \\
\hline \multicolumn{4}{|l|}{ TIMI flow } \\
\hline Grade 2 & $10(9.2)$ & $4(9.5)$ & \multirow{2}{*}{0.947} \\
\hline Grade 3 & $99(91)$ & $38(90)$ & \\
\hline De novo lesions at follow-up CAG & $16(15)$ & $4(9.5)$ & 0.402 \\
\hline Door-to-balloon time, minutes & $49(38-62)$ & $48(36-59)$ & 0.453 \\
\hline Total ischemic time, minutes & $178(105-368)$ & $204(133-372)$ & 0.232 \\
\hline \multicolumn{4}{|c|}{ Group according to total ischemic time (TIT) } \\
\hline$\leq 120$ minutes & $37(34)$ & $7(17)$ & 0.036 \\
\hline $120-150$ minutes & $10(9.2)$ & $7(17)$ & 0.192 \\
\hline $150-180$ minutes & $8(7.3)$ & $5(12)$ & 0.370 \\
\hline $180-210$ minutes & $7(6.4)$ & $4(9.5)$ & 0.511 \\
\hline$>210$ minutes & $47(43)$ & $19(45)$ & 0.814 \\
\hline \multicolumn{4}{|l|}{ Medications } \\
\hline Aspirin & $109(100)$ & $42(100)$ & - \\
\hline Clopidogrel & $92(84)$ & $31(74)$ & 0.133 \\
\hline Ticagrelor & $4(3.7)$ & $4(9.5)$ & 0.150 \\
\hline Prasugrel & $14(13)$ & $8(19)$ & 0.333 \\
\hline Cilostazol & $19(17)$ & $8(19)$ & 0.816 \\
\hline Statin & $109(100)$ & $42(100)$ & - \\
\hline$\beta$-Blockers & $93(85)$ & $37(88)$ & 0.659 \\
\hline ACE inhibitor & $24(22)$ & $11(26)$ & 0.586 \\
\hline Angiotensin II receptor blocker & $57(52)$ & $19(45)$ & 0.437 \\
\hline \multicolumn{4}{|l|}{ Laboratory assessments } \\
\hline Hemoglobin, g/dL & $14.4 \pm 2.1$ & $14.3 \pm 2.4$ & 0.759 \\
\hline $\mathrm{CRP}, \mathrm{mg} / \mathrm{dL}$ & $0.99 \pm 3.0$ & $0.55 \pm 1.5$ & 0.370 \\
\hline BUN, g/dL & $15.4 \pm 5.3$ & $14.8 \pm 5.8$ & 0.521 \\
\hline Creatinine, g/dL & $0.89 \pm 0.25$ & $0.88 \pm 0.28$ & 0.785 \\
\hline Glucose, mg/dL & $173 \pm 79$ & $161 \pm 48$ & 0.346 \\
\hline HbAlc, \% & $6.8 \pm 1.8$ & $6.4 \pm 1.2$ & 0.171 \\
\hline
\end{tabular}


TABLE 1: Continued.

\begin{tabular}{lccc}
\hline & DWMSI $\downarrow(n=109)$ & DWMSI $\uparrow(n=42)$ & $p$ value \\
\hline Total cholesterol, mg/dL & $177 \pm 45$ & $180 \pm 50$ & 0.723 \\
LDL, mg/dL & $118 \pm 49$ & $116 \pm 51$ & 0.860 \\
CK-MB, ng/mL & $147 \pm 110$ & $213 \pm 109$ & $\mathbf{0 . 0 0 1}$ \\
Troponin I, pg/mL & $30.4 \pm 34.3$ & $34.8 \pm 21.3$ & 0.440 \\
BNP, pg/mL & $104 \pm 199$ & $138 \pm 209$ & 0.382 \\
\hline
\end{tabular}

Values are presented as mean \pm standard deviation, $n$ (\%), or median (interquartile range). ACE, angiotensin-converting enzyme; BNP, brain natriuretic peptide; BUN, blood urea nitrogen; CAD, coronary artery disease; CAG, coronary angiography; CK-MB, creatine kinase-MB fraction; CRP, C-reactive protein; HbA1c, hemoglobin A1c; LDL, low-density lipoprotein; PCI, percutaneous coronary intervention; STEMI, ST-segment elevation myocardial infarction; TIMI, thrombolysis in myocardial infarction. Statistical significance was defined as $p<0.05$ by Welch's $t$-test (continuous variables) or the chisquared test (categorical variables). The values in bold indicate statistical significance $(p<0.05)$.

TABLE 2: Echocardiographic parameters.

\begin{tabular}{lccc}
\hline & $\Delta$ WMSI $\downarrow(n=109)$ & $\Delta$ WMSI $\uparrow(n=42)$ & $50.0 \pm 4.8$ \\
LV end-diastolic dimension, mm & $48.2 \pm 4.1$ & $34.0 \pm 6.3$ & $\mathbf{0 . 0 2 7}$ \\
LV end-systolic dimension, mm & $32.3 \pm 4.5$ & $9.8 \pm 1.1$ & 0.064 \\
Interventricular septum thickness, mm & $9.7 \pm 1.4$ & $9.9 \pm 1.2$ & 0.700 \\
LV posterior wall thickness, mm & $9.6 \pm 1.2$ & $52.5 \pm 8.7$ & 0.256 \\
LV ejection fraction, \% & $51.9 \pm 8.4$ & $33.9 \pm 9.0$ & 0.669 \\
LA volume index, ml/m ${ }^{2}$ & $33.4 \pm 11.4$ & $0.65 \pm 0.20$ & 0.812 \\
Early diastolic mitral inflow velocity (E), m/s & $0.59 \pm 0.19$ & $0.76 \pm 0.21$ & 0.101 \\
Late diastolic mitral inflow velocity (A), m/s & $0.74 \pm 0.18$ & $0.07 \pm 0.07$ & 0.465 \\
Mitral annulus early diastolic velocity (e'), m/s & $0.06 \pm 0.02$ & $12.6 \pm 5.9$ & 0.477 \\
E/ $e^{\prime}$ & $10.8 \pm 5.0$ & $25.8 \pm 10.1$ & 0.064 \\
RV systolic pressure, mm Hg & $25.5 \pm 8.5$ & 0.840 \\
\hline
\end{tabular}

Values are presented as mean \pm standard deviation. LA, left atrium; LV, left ventricle; RV, right ventricle. Statistical significance was defined as $p<0.05$ by Welch's $t$-test (continuous variables). The value in bold indicates statistical significance $(p<0.05)$.

Timely performance of the primary PCI as measured by door-to-balloon time has become one of the main quality measures in the treatment of patients with STEMI $[13,14]$. Several studies have failed to report improvements in mortality with shortened door-to-balloon times $[15,16]$. It is possible that further reductions in the mortality rate of STEMI patients in the modern era of primary PCI and adjuvant pharmacotherapy may be achieved only by means of reducing the TIT. Minimizing TIT is a major determinant of myocardial salvage, as the prolonged duration of ischemia is related to myocardial necrosis, as evidenced by cardiac magnetic resonance (CMR) imaging [17]. Some of the new imaging techniques, such as CMR and strain, are more precise for determining myocardial damage; however, they are less accessible techniques for daily practice. Some studies have reported a good correlation between WMSI and echocardiographic strain findings $[18,19]$. LV ejection fraction (LVEF) improves in some STEMI patients after effective reperfusion as a consequence of the gradual relief of myocardial stunning, whereas, in other patients, irreversible myocardial necrosis may result in chronic LV dysfunction [20]. Reverse LV remodeling occurred in a considerable proportion (37.7\%) of STEMI patients who underwent primary PCI, manifesting a poor prognosis $[20,21]$. The LVEF value is technique-dependent and may not accurately indicate the extent of myocardial damage due to regional compensatory effects [22-24]. On the other hand, it is estimated that an increase in the WMSI value within the first 12 to 24 hours after STEMI is a predictor of complications during hospital admission, such as malignant arrhythmia, pump failure, and mortality [25-27]. In addition, WMSI can be used to quantitatively measure the LV systolic function following STEMI [28].

Two studies reported a significant improvement in echocardiographic parameters, such as LVEF and WMSI, during follow-up after myocardial infarction [29, 30]. Such improved echocardiographic findings may indicate that welltimed and successful revascularization can restrict further myocardial remodeling. The results of follow-up echocardiography performed approximately three months after the episode suggested that, following PCI, future wall motion improvement is highly dependent on how quickly it is possible to reinstate perfusion in the occluded artery. In patients whose perfusion was restored earlier with PCI, the follow-up echocardiography reflected a significantly better degree of improvement [30]. The present study had a longer observation period than these two previous studies involving echocardiography and excluded cases of ISR through follow-up CAG; as such, we could discern the relationship between TIT and WMSI more clearly. Overall, in this study, the WMSI value tended to decrease regardless of TIT (Figure 2). However, patients who underwent primary PCI within 180 minutes experienced a more significant decrease in the WMSI value than those who underwent primary PCI after 180 minutes. This result suggests that there is an optimal TIT within which better outcomes in STEMI patients may be achieved.

In 1977, transient left circumflex coronary artery ligation was performed in dogs for different durations [31]. The 


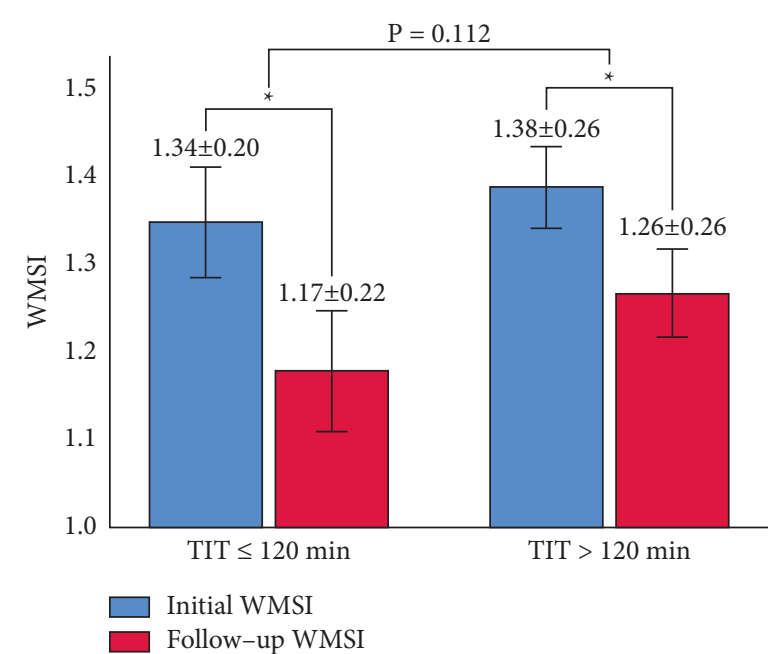

(a)

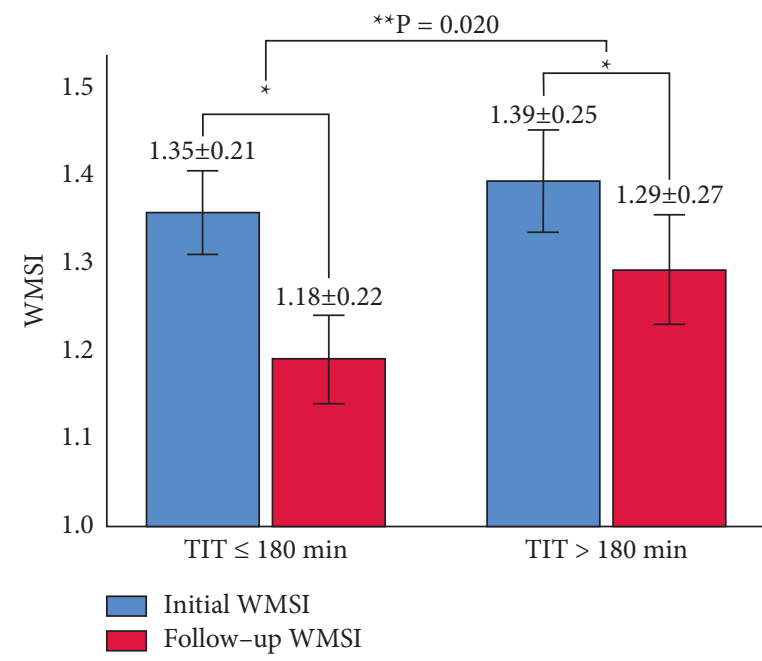

(c)

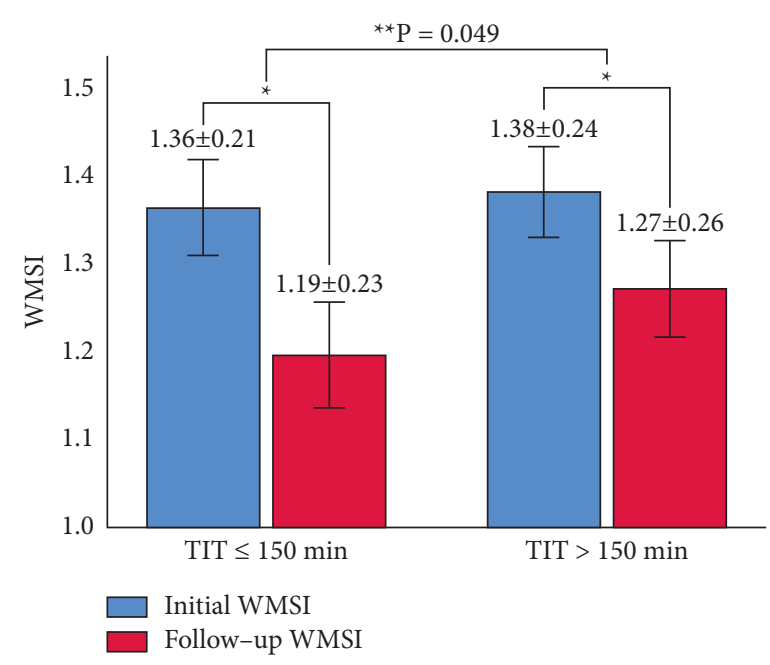

(b)

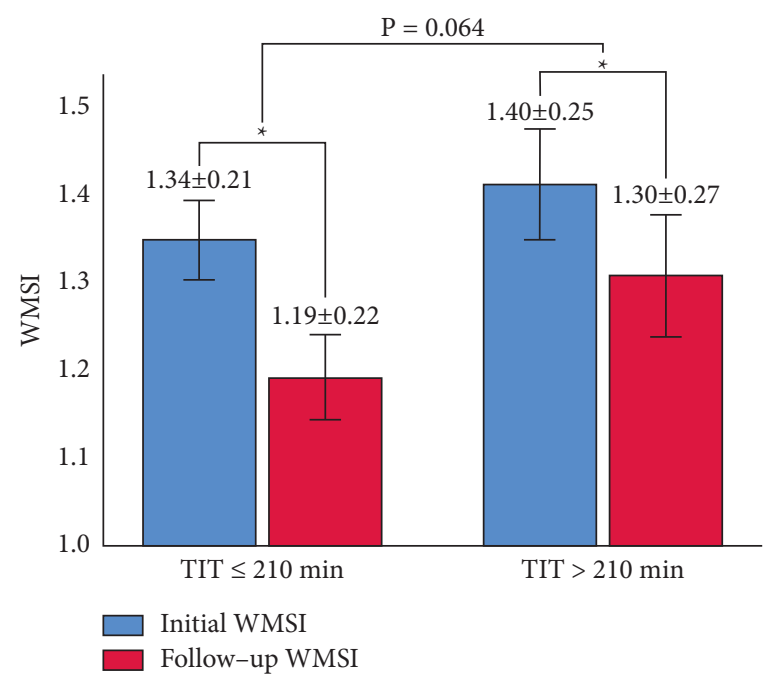

(d)

FIgURe 2: Changes in the WMSI value based on TITs of (a) 120, (b) 150, (c) 180, and (d) 210 minutes. TIT =total ischemic time; WMSI = wall motion score index.

TABLE 3: Variables associated with a decrease in the WMSI value among patients with TITs of 180 minutes or less $(N=74)$.

\begin{tabular}{lccc}
\hline & $\Delta$ WMSI $\downarrow(n=55)$ & $\Delta$ WMSI $\uparrow(n=19)$ & $p$ value \\
\hline Age & $61 \pm 14$ & $61 \pm 11$ & $14(74)$ \\
Male sex & $44(80)$ & $25.2 \pm 3.1$ & 0.971 \\
Body mass index & $24.2 \pm 2.8$ & $7(37)$ & 0.564 \\
Diabetes & $14(26)$ & $12(63)$ & 0.188 \\
Hypertension & $27(49)$ & $6(32)$ & 0.343 \\
Smoking ever & $26(47)$ & $7(37)$ & 0.290 \\
STEMI, anterior & $29(53)$ & $133(100-151)$ & 0.234 \\
Total ischemic time, minutes & $106(81-124)$ & $168 \pm 113$ & 0.232 \\
CK-MB & $134 \pm 103$ & $49.1 \pm 5.7$ & 0.244 \\
LV end-diastolic dimension, mm & $47.9 \pm 3.9$ & 0.301 \\
\hline
\end{tabular}

Values are presented as mean \pm standard deviation, $n$ (\%), or median (interquartile range). CK-MB, creatine kinase-MB fraction; LV, left ventricle; STEMI, ST-segment elevation myocardial infarction. Statistical significance was defined as $p<0.05$ by Welch's $t$-test (continuous variables) or the chi-squared test (categorical variables). The values in bold indicate statistical significance $(p<0.05)$.

percentage of transmural necrosis increased from $38 \%$ at 40 minutes of circumflex artery ligation duration to $85 \%$ at 24 hours of duration. Similar results have also been reported when evaluating cardiovascular outcomes in STEMI patients receiving fibrinolysis. For every 1,000 patients, 15 more lives were saved at one month of follow-up if the patients received 
TABLE 4: Predictor of a decrease in the WMSI value among patients with TITs of 180 minutes or less.

\begin{tabular}{lcccc}
\hline & Univariate & \multicolumn{2}{c}{${ }^{*}$ Multivariate } \\
& OR $(95 \% \mathrm{CI})$ & $p$ value & OR $(95 \% \mathrm{CI})$ & $p$ value \\
\hline Total ischemic time, minutes & $0.980(0.964-0.997)$ & 0.023 & $0.976(0.957-0.995)$ & 0.016 \\
\hline
\end{tabular}

*Adjusted for age, sex, body mass index, diabetes, hypertension, and smoking status.

treatment an hour earlier in the European Myocardial Infarction Project Group [32]. A meta-analysis of 22 randomized controlled trials studied more than 50,000 STEMI patients who received fibrinolysis. If fibrinolysis was achieved within one hour of symptom onset, there were 65 fewer deaths for every 1,000 patients when compared to patients who experienced greater delays from symptom onset to fibrinolysis [33]. Both animal and clinical data support the notion that shortening the duration of infarct artery occlusions can lead to smaller infarct sizes and lower mortality rates. However, the relationship is not linear and most of the benefit of reperfusion is seen within the first two hours of occlusion. In the CMR study, patients with a symptom-to-balloon time of greater than 121 minutes had significantly greater transmural necrosis, a larger infarct size, and decreased myocardial salvage [34]. The current guidelines recommend primary PCI as the preferred reperfusion strategy over thrombolysis in patients with STEMI, provided it can be performed within 120 minutes from STEMI diagnosis [10]. This would correspond with prolonged symptom-onset-to-balloon times of 3 to 4 hours. Of 1,791 patients with STEMI treated by primary angioplasty, a symptom-onset-to-balloon time of more than four hours was an independent predictor of one-year mortality [35]. In the Acute Coronary Syndrome Israeli Survey registry (involving 2,254 patients with STEMI treated by primary PCI), shortening of the TIT to less than 150 minutes was associated with improved long-term survival rates [36]. In the current study, based on a TIT of 180 minutes, a significant reduction in the WMSI value was experienced within a median followup period of 1 year. This result may explain why the optimal TIT exceeds two hours in clinical studies conducted in the modern reperfusion era of STEMI. There were significant changes in the WMSI value based on TITs of 150 and 180 minutes; especially considering a TIT of 180 minutes, TIT was an independent predictor of recovery from regional wall motion abnormalities. This study is meaningful in suggesting the criteria for the optimal TIT based on the WMSI value in STEMI patients.

4.1. Limitations. There are several limitations to this study. First, this was a retrospective study that was performed at a single academic hospital involving a relatively small sample population. However, all patients underwent follow-up CAG and had no ISR identified. In addition, this study had a relatively long observation period for recovery from regional wall motion abnormalities. Second, reporting bias is an inherent limitation of studying symptom-to-balloon time. Patients may find it difficult to accurately estimate the time of symptom onset, especially in the acute setting when suffering from pain and stress. Therefore, symptom-toballoon times may not have been independently verified.

\section{Conclusion}

In the modern reperfusion era of STEMI, patients with TITs of 180 minutes or less showed a significant degree of recovery from regional wall motion abnormalities compared to those with TITs of greater than 180 minutes. Every possible effort should be made to reduce TIT to 180 minutes or less, and further treatment for patients with TITs of greater than 180 minutes should be considered.

\section{Abbreviations}

CMR: Cardiac magnetic resonance

LV: $\quad$ Left ventricle

PCI: Percutaneous coronary intervention

STEMI: ST-segment elevation myocardial infarction

TIT: Total ischemic time

WMSI: Wall motion score index.

\section{Data Availability}

The data used to support the findings of this study are available from the corresponding author upon reasonable request.

\section{Disclosure}

This study was presented in the conference "The $72^{\text {nd }}$ Fall Conference of the Korean Society of Internal Medicine 2021.”

\section{Conflicts of Interest}

The authors declare no conflicts of interest.

\section{References}

[1] R. Estévez-Loureiro, Á. López-Sainz, and A. P. de Prado, "Timely reperfusion for ST-segment elevation myocardial infarction: effect of direct transfer to primary angioplasty on time delays and clinical outcomes," World Journal of Cardiology, vol. 6, no. 6, p. 424, 2014.

[2] B. Ibáñez, G. Heusch, M. Ovize, and F. Van de Werf, "Evolving therapies for myocardial ischemia/reperfusion injury," Journal of the American College of Cardiology, vol. 65, no. 14, pp. 1454-1471, 2015.

[3] A. Schömig, G. Ndrepepa, and A. Kastrati, "Late myocardial salvage: time to recognize its reality in the reperfusion therapy of acute myocardial infarction," European Heart Journal, vol. 27, no. 16, pp. 1900-1907, 2006. 
[4] A. H. A. s. A. M. I. A. W. Group, A. K. Jacobs, and E. M. Antman, "Recommendation to develop strategies to increase the number of ST-Segment-Elevation Myocardial Infarction patients with timely access to primary percutaneous coronary intervention," Circulation, vol. 113, no. 17, pp. 2152-2163, 2006.

[5] D. Kawecki, B. Morawiec, M. Gąsior, K. Wilczek, E. Nowalany-Kozielska, and M. Gierlotka, "Annual trends in total ischemic time and one-year fatalities: the paradox of STEMI network performance assessment," Journal of Clinical Medicine, vol. 8, no. 1, p. 78, 2019.

[6] K. A. Reimer and R. B. J. L. i. Jennings, "a. j. o. t. methods, and pathology, "The" wavefront phenomenon" of myocardial ischemic cell death. II. Transmural progression of necrosis within the framework of ischemic bed size (myocardium at risk) and collateral flow," Laboratory Investigation, vol. 40, no. 6, pp. 633-644, 1979.

[7] K. T. Ahn, Y. B. Song, Y. H. Choe et al., "Impact of transmural necrosis on left ventricular remodeling and clinical outcomes in patients undergoing primary percutaneous coronary intervention for ST-segment elevation myocardial infarction," The International Journal of Cardiovascular Imaging, vol. 29, no. 4, pp. 835-842, 2013.

[8] L. Nechvatal, O. Hlinomaz, L. Groch et al., "Serial echocardiographic assessment of the left ventricular function after direct PCI," Kardiologia Polska, vol. 59, no. 11, pp. 397-401, 2003.

[9] S. A. Wickline, E. D. Verdonk, A. K. Wong, R. K. Shepard, and J. G. Miller, "Structural remodeling of human myocardial tissue after infarction. Quantification with ultrasonic backscatter," Circulation, vol. 85, no. 1, pp. 259-268, 1992.

[10] B. Ibanez, S. James, and S. Agewall, "2017 ESC Guidelines for the management of acute myocardial infarction in patients presenting with ST-segment elevation: the Task Force for the management of acute myocardial infarction in patients presenting with ST-segment elevation of the European Society of Cardiology (ESC)," European Heart Journal, vol. 39, no. 2, pp. 119-177, 2018.

[11] H. Jneid, D. Addison, and D. L. Bhatt, "2017 AHA/ACC clinical performance and quality measures for adults with STelevation and non-ST-elevation myocardial infarction: a report of the American College of Cardiology/American Heart Association Task Force on Performance Measures," Circ Cardiovasc Qual Outcomes, vol. 10, no. 10, Article ID e000032, 2017.

[12] R. M. Lang, L. P. Badano, V. Mor-Avi et al., "Recommendations for cardiac chamber quantification by echocardiography in adults: an update from the American Society of Echocardiography and the European Association of Cardiovascular Imaging," European Heart Journal - Cardiovascular Imaging, vol. 16, no. 3, pp. 233-271, 2015.

[13] A. T. F. Members, P. G. Steg, and S. K. James, "ESC Guidelines for the management of acute myocardial infarction in patients presenting with ST-segment elevation: the Task Force on the management of ST-segment elevation acute myocardial infarction of the European Society of Cardiology (ESC)," European Heart Journal, vol. 33, no. 20, pp. 2569-2619, 2012.

[14] P. T. O'gara, F. G. Kushner, and D. D. Ascheim, "2013 ACCF/ AHA guideline for the management of ST-elevation myocardial infarction: a report of the American college of Cardiology foundation/American heart association task force on practice guidelines," Journal of the American College of Cardiology, vol. 61, no. 4, pp. e78-e140, 2013.
[15] A. Lubovich, I. Dobrecky-mery, E. Radzishevski et al., "Bypassing the emergency room to reduce door-to-balloon time and improve outcomes of ST elevation myocardial infarction patients: analysis of data from 2004-2010 ACSIS registry," Journal of Interventional Cardiology, vol. 28, no. 2, pp. 141-146, 2015.

[16] D. S. Menees, E. D. Peterson, Y. Wang et al., "Door-to-balloon time and mortality among patients undergoing primary PCI," New England Journal of Medicine, vol. 369, no. 10, pp. 901909, 2013.

[17] G. Tarantini, L. Cacciavillani, F. Corbetti et al., "Duration of ischemia is a major determinant of transmurality and severe microvascular obstruction after primary angioplasty," Journal of the American College of Cardiology, vol. 46, no. 7, pp. 1229-1235, 2005.

[18] C. Eek, B. r. Grenne, H. Brunvand et al., "Strain echocardiography and wall motion score index predicts final infarct size in patients with non-ST-segment-elevation myocardial infarction," Circulation: Cardiovascular Imaging, vol. 3, no. 2, pp. 187-194, 2010.

[19] N. Mistry, J. O. Beitnes, S. Halvorsen et al., "Assessment of left ventricular function in ST-elevation myocardial infarction by global longitudinal strain: a comparison with ejection fraction, infarct size, and wall motion score index measured by non-invasive imaging modalities," European Journal of Echocardiography, vol. 12, no. 9, pp. 678-683, 2011.

[20] G. W. Serrao, A. J. Lansky, R. Mehran, and G. W. Stone, "Predictors of left ventricular ejection fraction improvement after primary stenting in ST-segment elevation myocardial infarction (from the harmonizing outcomes with revascularization and stents in acute myocardial infarction trial)," The American Journal of Cardiology, vol. 121, no. 6, pp. 678-683, 2018.

[21] J. C. Choe, K. S. Cha, E. Y. Yun et al., "Reverse left ventricular remodelling in st-elevation myocardial infarction patients undergoing primary percutaneous coronary intervention: incidence, predictors, and impact on outcome," Heart Lung \& Circulation, vol. 27, no. 2, pp. 154-164, 2018.

[22] J. Berning and F. Steensgaard-Hansen, "Early estimation of risk by echocardiographic determination of wall motion index in an unselected population with acute myocardial infarction," The American Journal of Cardiology, vol. 65, no. 9, pp. 567-576, 1990.

[23] X. Chen, F. Liu, H. Xu et al., "Left ventricular diastolic dysfunction in patients with ST-elevation myocardial infarction following early and late reperfusion by coronary intervention," International Journal of Cardiology, vol. 228, pp. 886-889, 2017.

[24] J. Oh, "Echocardiography in heart failure: beyond diagnosis," European Journal of Echocardiography, vol. 8, no. 1, pp. 4-14, 2007.

[25] Y. Neuman, B. Cercek, J. Aragon et al., "Comparison of frequency of left ventricular wall motion abnormalities in patients with a first acute myocardial infarction with versus without left ventricular hypertrophy," The American Journal of Cardiology, vol. 94, no. 6, pp. 763-766, 2004.

[26] C. M. Otto, The Practice of Clinical Echocardiography, Elsevier Health Sciences, Amsterdam, Netherlands, 2007.

[27] N. Witt, B. A. Samad, M. Frick, M. Alam, and f. imaging, "Detection of left ventricular dysfunction by Doppler tissue imaging in patients with complete recovery of visual wall motion abnormalities 6 months after a first ST-elevation myocardial infarction," Clinical Physiology and Functional Imaging, vol. 27, no. 5, pp. 305-308, 2007. 
[28] N. B. Schiller, P. M. Shah, M. Crawford et al., "Recommendations for quantitation of the left ventricle by two-dimensional echocardiography," Journal of the American Society of Echocardiography, vol. 2, no. 5, pp. 358-367, 1989.

[29] S. Hadi, M. Toufan Tabrizi, and A. Separham, "Prevalence and predictors of left ventricle regional wall motion abnormality 6 Weeks after primary percutaneous intervention in patients with first acute anterior myocardial infarction," Crescent $J$ Med Biol Sci, vol. 7, no. 1, 2020.

[30] I. Rácz, L. Fülöp, R. Kolozsvári et al., "Wall motion changes in myocardial infarction in relation to the time elapsed from symptoms until revascularization," The Anatolian Journal of Cardiology, vol. 15, no. 5, pp. 363-70, 2015.

[31] K. A. Reimer, J. E. Lowe, M. M. Rasmussen, and R. B. Jennings, "The wavefront phenomenon of ischemic cell death. 1. Myocardial infarct size vs. duration of coronary occlusion in dogs," Circulation, vol. 56, no. 5, pp. 786-794, 1977.

[32] E. M. I. P. G. J. N. E. J. o. Medicine, "Prehospital thrombolytic therapy in patients with suspected acute myocardial infarction," New England Journal of Medicine, vol. 329, no. 6, pp. 383-389, 1993.

[33] E. Boersma, A. C. Maas, J. W. Deckers, and M. L. J. T. L. Simoons, "Early thrombolytic treatment in acute myocardial infarction: reappraisal of the golden hour," Lancet, vol. 348, no. 9030, pp. 771-775, 1996.

[34] S. Greulich, A. Mayr, and S. Gloekler, "Time-dependent myocardial necrosis in patients with ST-segment-elevation myocardial infarction without angiographic collateral flow visualized by cardiac magnetic resonance imaging: results from the multicenter STEMI-SCAR project," Journal of American Heart Association, vol. 8, no. 12, Article ID e012429, 2019.

[35] G. De Luca, H. Suryapranata, J. P. Ottervanger, and E. M. Antman, "Time delay to treatment and mortality in primary angioplasty for acute myocardial infarction," Circulation, vol. 109, no. 10, pp. 1223-1225, 2004.

[36] A. Lubovich, E. Radzishevsky, I. Goldenberg, S. Matezky, and U. J. J. I. C. Rosenschein, "Total ischemic time and short, intermediate and long term mortality of patients with STEMI treated by primary percutaneous coronary intervention: analysis of data from 2004-2013 ACSIS registry," J Integr Cardiol, vol. 4, pp. 1-4, 2018. 